\title{
Active bio contact mechanics: Concepts of active control of wear and growth of the cartilage in natural joints
}

Cite as: AIP Conference Proceedings 2167, 020285 (2019); https://doi.org/10.1063/1.5132152

Published Online: 19 November 2019

V. L. Popov

\section{ARTICLES YOU MAY BE INTERESTED IN}

Regimes of adhesive wear in dry contact: Conditions of realization and determining parameters

AIP Conference Proceedings 2167, 020075 (2019); https://doi.org/10.1063/1.5131942

Adhesive contribution to friction

AIP Conference Proceedings 2167, 020286 (2019); https://doi.org/10.1063/1.5132153

Mechanics of adhesive contacts: Experiment and theory

AIP Conference Proceedings 2167, 020201 (2019); https://doi.org/10.1063/1.5132068
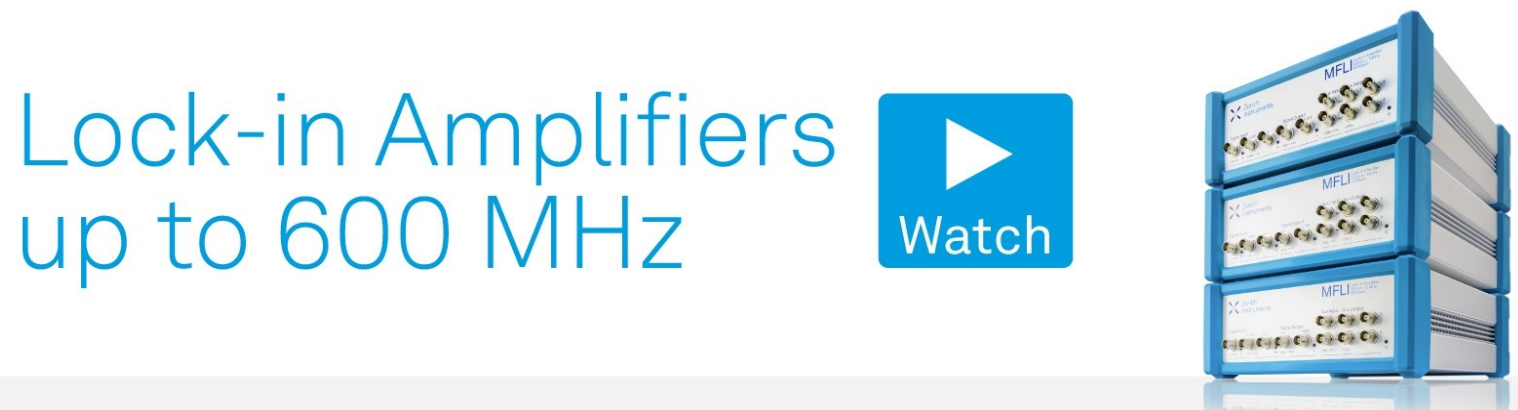


\title{
Active Bio Contact Mechanics: Concepts of Active Control of Wear and Growth of the Cartilage in Natural Joints
}

\author{
V.L. Popov ${ }^{\mathrm{a})}$ \\ Technische Universität Berlin, Berlin, 10623 Germany \\ ${ }^{a)}$ Corresponding author: v.popov@tu-berlin.de
}

\begin{abstract}
The modern practice of treating patients with impaired motor functions often consists in replacing the natural joints with artificial ones. Currently, more than 1.5 million hip joint replacements are performed annually in the world, with a tendency to grow. Even if the replacement procedure is successful, replacement is often ineffective in the medium or long term, due to various complications. The paper puts forward ideas and discusses how to mechanically load the joint to promote the development of regenerative effects, while minimizing the influence of factors determining wear.
\end{abstract}

\section{INTRODUCTION}

Moving parts, independently of whether natural or artificial, are subject to wear. In the case of natural joints, the wear can finally lead to disfunctions of the joint. One of the possible solutions is the joint replacement [1]. Currently, more than 1.5 million hip joint replacements are performed annually in the world, with a tendency to grow [2]. In many cases, after surgery, the patient may return to an active lifestyle for a certain time, although arthroplasty always leads to a reduction in joint mobility. At the same time, in a small, but significant percentage of cases, the implantation of an artificial joint leads to strong immune reactions, which practically do not allow the patient to return to a normal life. Even if the replacement procedure is successful, replacement is often ineffective in the medium or long term, due to various complications. Complications are of a different nature and may be associated with a significant decrease in the setting between the implant and the bone, wear of the implant, wear and thermally induced necrosis. All these processes may ultimately cause immune reactions at the organism level. The occurrence of complications leads to the need for new surgical interventions, which require not only large financial costs but above all lead to the additional physical suffering of patients and long-term postoperative recovery. Of particular concern is the current tendency to reduce the average age of patients who need joint replacement for medical reasons. Reducing the age of patients leads to the fact that repeated surgical interventions become almost inevitable. It is important to note that the result of secondary endoprosthetics in comparison with the primary one is always often unpredictable.

The necessity and importance of endoprosthetics in cases of significant damage to the joints, or with severe wear or partial destruction of the cartilage layer, as well as its damage during prolonged immobility, is beyond doubt. However, at the initial stage of the disease and deterioration of the mechanical properties of the joint, it is natural to ask what measures can be taken to correct the situation and allow the joint to regenerate itself. It is known that under certain conditions, the cartilage layer is able to regenerate. The process of regeneration of cartilage tissue is extremely slow and depends on many factors, such as the type of damage. The key role in regeneration is played by the type of mechanical loading of the articular joint. Mechanical loading plays a dual role. On the one hand, the load is necessary to maintain the normal functioning of the cartilage. Cartilage tissue does not contain blood vessels. The nutrition of cartilage cells is due to the diffusion of nutrients and oxygen from the synovial fluid located in the interarticular space. To activate these diffusion processes, periodic mechanical loading of the joint is necessary. On the other hand, excessive mechanical loading can lead to wear and discontinuities in the cartilage body. 
Therefore, the topical question is how to load the joint to promote the development of regenerative effects, while minimizing the influence of factors determining wear. During the introductory plenary presentation at the World Tribology Congress 2017 in Beijing, Jacob Klein (Weizmann Institute of Science, Israel) formulated a simple qualitative principle of the anabolic (restorative) or catabolic (wear) response of the cartilage body to stress: normal cartilage load contributes to their growth, and tangential load leads to wear.

The meaning of this rule of thumb is simple. It is well known, for example, from the Hertz contact theory that a normal load leads to an increase in the diagonal components of the stress tensor, which determine the increase in hydrostatic pressure. At the same time, shear stresses that have a negative effect on the joint are quite small. The pressure gradient during normal loading has the "pump effect" and promotes the diffusion of extracellular fluid from the deep layers of cartilage to the contact area. Tangential stresses, on the other hand, are directly dangerous loads for the material of the joint, and do not lead to the "pump effect". In addition, tangential stresses in the "rear" part of the contact cause the occurrence of tensile stresses in the material. The development of such stresses is the cause of fracture of the cartilage tissue. From what has been said, it is obvious that periodic normal loads contribute to the normal functioning of cartilage tissue, while the tangential ones should be minimized. The implementation of this simple idea needs consideration of detailed contact mechanics of joint under various load conditions.

\section{MECHANICS OF THE JOINT CARTILAGE TISSUE}

In the joint, two surfaces are in contact being formed of hard bone tissues covered with a softer tissue, the joint cartilage. Early analytical models of cartilage tissue [3] describe the contact problem of layered elastic bodies and analyze the influence of the cartilage thickness and stiffness on the resulting contact stresses. Later, more complicated models for cartilage tissue have been proposed including two-phase (and even three-phase), viscoelastic, poro-viscoelastic [4] and microstructural ones, which take into account the effect of chondrocyte cells [5] and collagen networks [7].

Despite the fact that the finite element method is a powerful and flexible tool for modeling mechanical systems with complex three-dimensional geometry and different rheological properties, it is still extremely time-consuming, especially when solving contact problems. This is due to highly localized stress distribution as well as a priori unknown contact area and boundary conditions. Various analytical asymptotic methods (a comprehensive review of such methods is given in [8]) are created as an alternative to the description of cartilages. Rapid numerical simulations methods for elastic and viscoelastic contacts are based on the Boundary Element Method [9].

\section{MECHANICALLY INDUCED AND DRUG SUPPORTED GROWTH OF CARTILAGE}

The first fundamental work that studies the mechanisms of cell division under loading was devoted to the study of bone growth and was published more than 100 years ago [10]. To date, a large amount of the experimental data on the restructuring and development of the cellular structure of various organs under external forces has been accumulated. The available data clearly indicate that periodic loadings lead to cell structure regeneration [11,12], while static loads do not lead to such an effect. Clinical studies show that the articular cartilage thickness decreases from 9 to 13 percent in the absence of a normal load for one year [13]. It is important to note that the benefits of mechanical stress on the joint cartilage have short-term and long-term efficiency. The studies $[14,15]$ show that moderate physical loading on the joints of animals can increase the thickness of cartilage and proteoglycans.

Tissue growth is a complex nonlinear process depending on many factors. The external load is a controlling parameter that sets the modes of repair and cellular reorganization of tissues. A theory was suggested according to which the modes of bone tissue growth depend on the deformation level in it [16].

The growth process under loading often proceeds as follows. First, cells that will form a new tissue enter the zone that is under mechanical loading [17]. Further, depending on the load type, the embryocells are transformed into one or another tissue type. A certain phase diagram was constructed in [17], which includes the growth areas of various tissue types, among them cartilage one.

Cartilage tissue differs from other tissues in a smaller proportion of cells that are surrounded by an extracellular substance, being a source of nutrition. The main components of cartilage are chondrocytes (cartilage cells) and the extracellular matrix made of fibers. The amount of chondrocytes is about 1 percent of the total mass of the cartilage in an adult [18]. Due to a low content of chondrocytes, cartilage repair processes, as a rule, take much time. The hip joint cartilage contains more than 70 percent of water. The water is bound by the hyaluronic acid, which provides 
elastic properties to cartilage. All measures to repair the cartilage are mainly aimed at increasing the number of chondrocytes, which form a new cartilage tissue.

There are several natural mechanisms for repairing the cartilage. The first is the growth of a new layer of cartilage cells due to their division. In addition, the synovial membrane of the joint grows in the case of significant injuries, finally turning into a tissue similar to cartilage. Transformation of bone cells into cells with properties similar to cartilage ones is also possible, but also a reverse process can occur [19]. Sometimes surface cartilage damage can be fully restored using special medications (somatotropic hormone, insulin, thyrocalcitonin, and anabolic steroids). Somatotropic hormone stimulates the division of chondrocytes [20], and chondrocytes restore the matrix. However, this drug induces the cartilage growth throughout the body, and not just where it is needed. Therefore, its use without extreme necessity is undesirable. Since in the zone of intense mechanical stress the cartilage grows much faster under the action of this drug, it is possible to use a combined load mode and the drug in small doses.

Mechanical loading triggers the process of chondrocyte division, and it can be applied exactly to the damaged joint, which is preferable to somatotropic hormone. There are many papers studying the effect of mechanical stress on the regeneration of cartilage tissue. For example, the work [21] indicates that both reparative and destructive processes occur in cartilage under loading, and the general cartilage evolution is determined by the rate of these processes. In this regard, the optimal loading mode should be chosen; to search for it is one of the key objectives of the future research.

Current investigations on the influence of various factors are often performed on animals [22]. In particular, it was found that static compression inhibits the synthesis of proteoglycans and proteins, and dynamic compression significantly stimulates the production of the matrix, and these are dependent on the frequency and amplitude of the loading [23]. Dynamic compression induces cell and matrix deformation, the appearance of a pressure gradient in tissues and fluid flow, and as a result leads to the transport of macromolecular cytokines, growth factors, enzymes, and other substances. In contrast, shear deformation in the cartilage does not cause volume changes and does not lead to fluid flow and pressure gradient [24]. Here we come back to the ideas stated already in the introduction. In the next Section we illustrate this idea with a simple numerical simulation.

\section{A NUMERICAL MODEL FOR WEAR AND GROWTH OF JOINT MATERIAL}

Wear process is mostly described by a simple law stating that the wear volume, $V$, is given by the equation

$$
V=k_{\text {adh }} \frac{F_{N} x}{\sigma_{0}},
$$

where $F_{N}$ is the normal load, $x$ is the sliding distance, $\sigma_{0}$ the hardness of contacting materials (or the smaller one if they are different), and $k_{\text {adh }}$ is the coefficient of adhesive wear. This equation is often called "Archard's law" [25]. On the other hand, dynamic loadings producing pressure gradient can stimulate the cartilage growth (which can be considered as a "negative wear"). In this study, just for illustrative purposes, we assume that the growth rate is proportional to the changing rate of the pressure gradient:

$$
\dot{\mathrm{V}} \propto \sqrt{\left\langle\left(\frac{\partial \dot{\mathrm{p}}}{\partial \mathrm{z}}\right)^{2}\right\rangle},
$$

where $p$ is hydrostatic pressure and $z$ normal coordinate and $\langle\ldots\rangle$ means averaging over one oscillation period.

In the Fig. 1 we illustrate the action of tangential and normal loading on the changes of the surfaces of joint contact (for simplicity, all changes are shown only on one partner). Tangential loading leads to a typical flattening due to wear under conditions of gross slip [27] (Fig. 1a). Tangential loading produce practically no pressure gradients, so the growth processes are not activated. The pure normal loading lead practically to no wear but produces essential pressure gradients leading to regenerative processes (Fig. 1b). 
Tangential loading

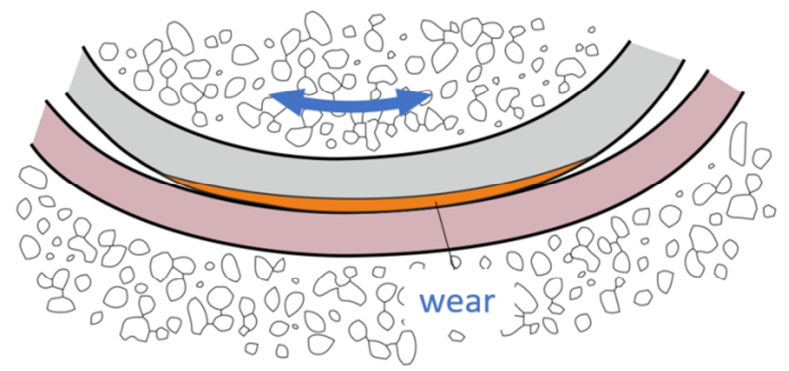

(a)

\section{Normal loading}

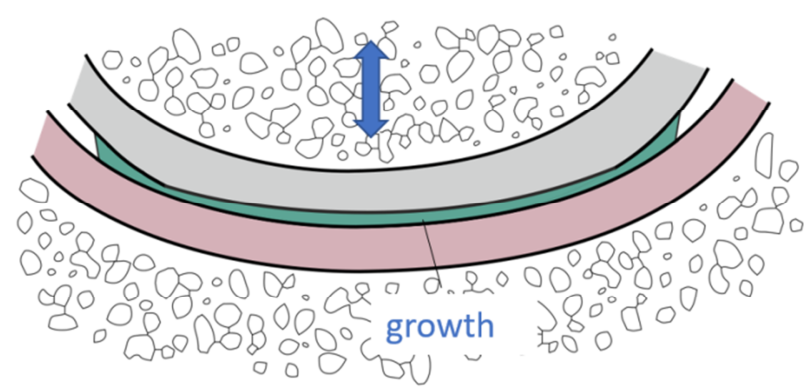

(b)

FIGURE 1. Schematic presentation of wear under tangential loading and growth under normal loading.

In the Fig. 2, we present results of numerical simulations of the growth process under normal oscillation. The pressure gradient was calculated using the Method of Dimensionality Reduction [26] directly on the contact surface. The distribution of the pressure gradient is shown in the lower part of Fig. 2. It is seen that it is concentrated immediately outside the boundary of the contact. This leads to propagation of the layer and closing the existing gap. We suggest that this may be the mechanism of healing also of other types of defects of the cartilage layer.
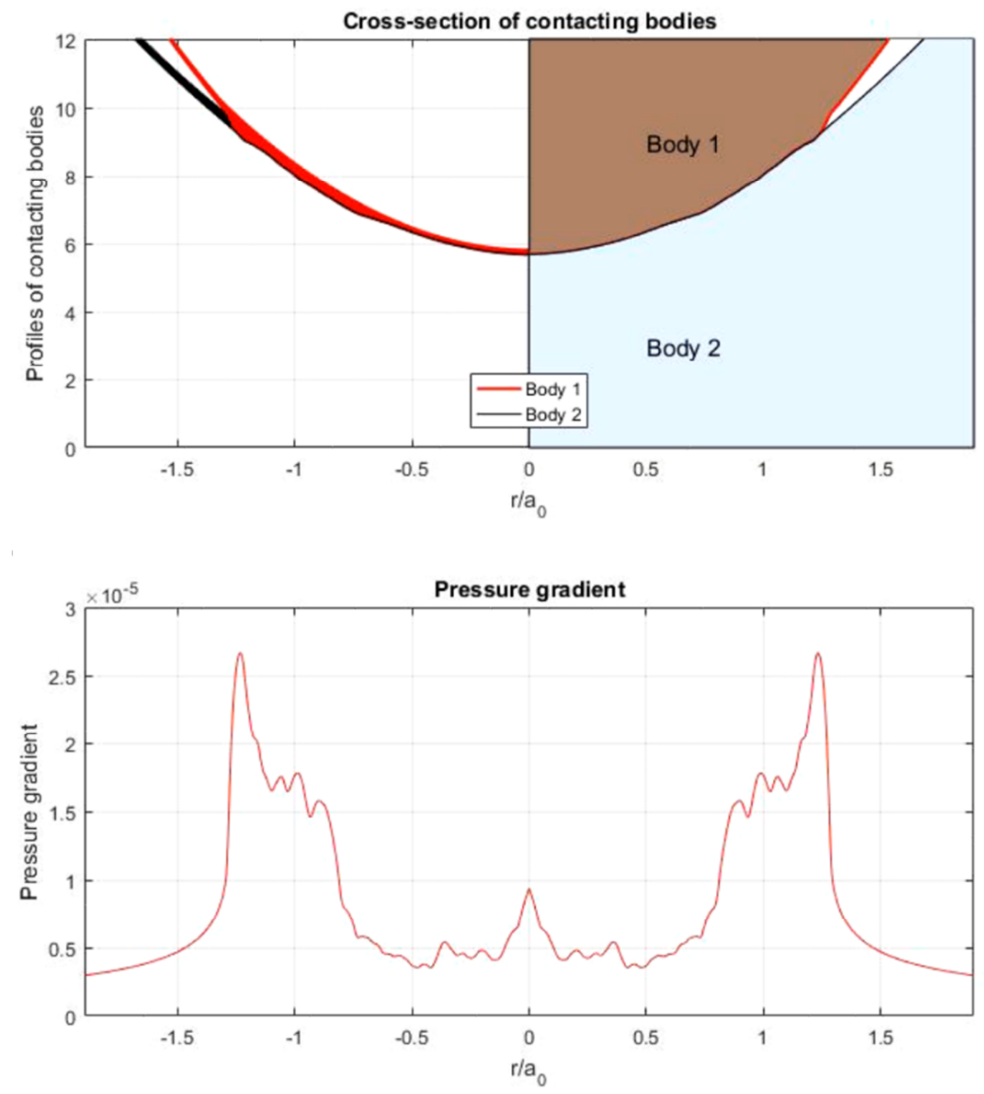

FIGURE 2. Growth of cartilage layer simulated based on the rule (2). On the right side of the upper subfigure, the configuration of contact is shown, while the left side shows the changes in the surface configuration (thick read "line" shows the change of the shape due to growth processes). The lower figure shows the distribution of the pressure gradient. 


\section{CONCLUSION}

The multidisciplinary nature of the problem of regeneration of natural joints makes necessary further intensive research, both experimental and theoretical. On the theoretical side, construction of a new, coupled mathematical models is needed which have to take into account the features of the contact interaction of surface layers of cartilage tissue in the presence of synovial fluid. The model has to include wear processes, complex viscoelastic mechanical properties of the cartilage tissue, diffusion processes within the cartilage tissue, migration of the cells and the effect of mechanical stresses on them and cartilage regeneration processes.

\section{ACKNOWLEDGMENTS}

The author acknowledges inspiring discussions with S. Psakhie, I. Lyashenko, E. Willert, E. Shilko, V. Pakhaliuk, A. Poliakov, F. Forsbach, Z. Wang, and H. Zhang. The author is deeply thankful to Qiang Li for his support in numerical simulations.

\section{REFERENCES}

1. I. D. Learmonth, C. Young, and C. Rorabeck, Lancet 370, 1508-19 (2007).

2. J. M. Wilkinson, "Why do implants fail?," Presented at: 15th EFORT Congress: a combined programme in partnership with the BOA, London, 4-6 June, 2014.

3. A.W. Eberhardt et al., J. BioMech. Eng. 112, 407-413 (1990).

4. W. Wilson et al., J. BioMech. 38, 1195-1204 (2015).

5. S. Federico et al., J. BioMech. 38, 2008-2018 (2005).

6. P. Bursać et al., J. BioMech. Eng. 122, 347-353 (2000)

7. G.A. Ateshian et al., J. BioMech. 48, 779-786 (2015).

8. I. Argatov and G. Mishuris, Front Bioeng Biotechnol 4, 83 (2016).

9. R. Pohrt and Q. Li, Physical Mesomechanics 17 (4), 334-340 (2014).

10. J. Wolff, Das Gesetz der Transformation der Knochen (Verlag von August Hirschwald, Berlin, 1892).

11. M. Van der Meulen and R. Huiskes, J. Biomech 35, 401- 414 (2002).

12. A.I. Volozhin and V.S. Oganov (Eds.), Osteoporosis (Practical Medicine, Moscow, 2005). (in Russian)

13. B. Vanwanseele, F. Eckstein, H. Knecht, A. Spaepen, and E. Stüssi, Arthritis Rheum. 48, 3377-3381 (2003)

14. I. Kiviranta et al., J Orthop Res. 6, 188-95 (1988).

15. J. Jurvelin et al., Int J Sports Med 7, 106-110 (1986).

16. F. Pauwels, "A new theory concerning the influence of mechanical stimuli on the differentiation of the supporting tissue" in Biomechanics of the locomotor apparatus, edited by P. Maquet, R. Furlong (Springer, 1980)

17. L.B. Maslov, Russian Journal of Biomechanics 17, 39-63 (2013).

18. G.P. Kotelnikov, Osteoarthritis of the hip and knee joints (GEOTAR-Media, Moscow, 2012) (in Russian).

19. Yu.B. Bulanov, Muscle Nutrition Review 8, 35-42 (2002).

20. K. Madsen et al., Nature 304 (5926), 545-7 (1983).

21. A.P. Kurpyakov, "Experimental models for investigating the damage and repair of the articular cartilage of the synovial joints," Ph.D. thesis, FSBI "RCME named after N.N. Priorov", 2009.

22. S.P. Mironov et al., N.N. Priorov, Journal of Traumatology and Orthopedics 1, 57-63 (2006). (in Russian)

23. R.L. Sah et al., J Orthop Res. 7(5), 619-36 (1989).

24. A.J Grodzinsky et al., Annu Rev Biomed Eng. 2, $691-713$ (2000).

25. J. F. Archard, J. Appl. Phys. 24, 981 (1953).

26. V.L. Popov and M. Heß, Method of dmensionality reduction in contact mechanics and friction (Springer, 2015)

27. V.L. Popov, M. Heß, and E. Willert, Handbuch der Kontaktmechanik (Springer, 2017) 